\title{
Sedimentation of Suspensions in Shear-Thinning Fluids
}

\author{
S. Daugan 1, 2, L. Talini ${ }^{1}$, B. Herzhaft ${ }^{2}$, Y. Peysson ${ }^{2}$ and C. Allain ${ }^{*}$ \\ 1 Laboratoire FAST, Bât. 502, Campus Universitaire, 91405 Orsay Cedex - France \\ 2 Institut français du pétrole, 1 et 4, avenue de Bois-Préau, 92852 Rueil-Malmaison Cedex - France \\ e-mail: benjamin.herzhaff@ifp.fr - yannick.peysson@ifp.fr \\ ${ }^{*}$ Corresponding author: allain@fast.u-psud.fr
}

\begin{abstract}
Résumé - Sédimentation de particules dans un fluide rhéofluidifiant - Cet article présente l'étude de la sédimentation de suspensions de particules monodisperses et non browniennes dans des solutions polymères rhéofluidifiantes. Contrairement à ce qui est observé en milieu newtonien, la sédimentation dans ce type de fluide n'est pas caractérisée par l'apparition d'un front de sédimentation ; l'agrégation des particules conduit de plus à la formation de structures verticales riches en particules ainsi qu'à une répartition inhomogène des particules le long de la hauteur de la cellule. Une fois ces structures formées, la suspension s'appauvrit rapidement en particules puis la sédimentation devient très lente aux temps longs. Nous montrons que les variations temporelles de la fraction volumique moyenne en particules à une hauteur donnée peut être décrite par deux temps caractéristiques. Nous avons étudié les variations de ces temps en fonction de la concentration en polymère et de la fraction volumique initiale en particules. Contrairement aux résultats bien connus en milieu newtonien, plus la suspension est concentrée en particules, plus celle-ci s'appauvrit rapidement. En raison des phénomènes très lents intervenant aux temps longs, le temps nécessaire pour séparer complètement les particules du fluide peut toutefois être supérieur à celui en milieu newtonien. Enfin, nous présentons une étude de la structuration spatiale des suspensions en fonction des différents paramètres.
\end{abstract}

\begin{abstract}
Sedimentation of Suspensions in Shear-Thinning Fluids - We present an experimental study of the sedimentation of non-Brownian monodisperse suspensions in polymeric shear-thinning fluids. In such media and contrary to what is observed in Newtonian fluids, no sedimentation front appears; particle aggregation moreover leads to the formation of vertical particle-rich structures and of an inhomogeneous particle volume fraction along the cell height. Once these structure formed, a very efficient settling occurs whereas the process further slows down at long times. We show that the temporal variations of the mean volume fraction at a given height can be described using two characteristic times. We have systematically studied the evolution of these times with the polymer concentration and the particle volume fraction of the suspension. Contrary to the well known results in Newtonian fluids, the more concentrated in particles is the suspension, the more efficient is the settling within intermediate times. The time needed for a complete separation of fluids and particles can however be longer than in a Newtonian fluid owing to the slow variations of the volume fraction at long times. Finally, the formation of the vertical structures is also studied according to the different parameters.
\end{abstract}




\section{INTRODUCTION}

The use of rotary drilling technique since the beginning of the $20^{\text {th }}$ century increases considerably the depth of the oil wells compared with the previous hammering technique. The injection of a fluid while drilling improves rock cuttings removal and in the same time increases the mechanical stability of the hole. Water was used at first, but now drilling fluids are complex fluids composed of a lot of different components. Indeed, several properties have to be controlled to correctly drill wells (viscosity, density, stability, etc.). Polymer, chemical additives, solid powder, etc are used to enhance and control the properties of those fluids which are highly non-Newtonian. Drilling fluids are usually shear-thinning and can be described as power law fluids. When the circulation is stopped, the fluid is at rest and the sedimentation of the drilling cutting must be limited. As a consequence, the problem of settling of particles in those non-Newtonian fluids and specifically shear thinning fluid is of great importance.

From a more fundamental point of view the settling behavior in non-Newtonian fluids has been the subject of only a few experimental studies and remains poorly known. Most studies focus on the settling of a single sphere (see [1] for a detailed review) or of a few isolated particles [2-5]. However, the large variety of rheological behaviors exhibited by polymeric fluids prevents one from generalizing the obtained results. The case of suspensions has been even more poorly studied. Studies in either shear-thinning $[6,7]$ or elastic [7] fluids show the development of nonhomogeneous structures during sedimentation that are induced by particle aggregation. Moreover, the sedimentation rate in such fluids is not constant: the suspensions first settle rapidly and the process later grows slower in time [7]. There is however a lack of quantitative results to characterize the different settling regimes as well as the apparition of structures within the suspensions.

Herein, we present a study of the sedimentation of monodisperse suspensions in fluids that are strongly shearthinning and present small normal stresses differences. We have shown in previous studies dealing with the coaxial settling of a few particles that particles are strongly prone to aggregate in such fluids [8-10]. We have in particular demonstrated the existence of a critical separation distance between particles such that the particles form a stable cluster when a distance smaller than this critical one initially separates them. This aggregation behavior results from the creation of a corridor of reduced viscosity in the wake of the first particle that shears the fluid. This corridor vanishes when the fluid relaxes and the existence of a critical separation distance is the consequence of the fluid healing after the passage of the first particle and can be linked to the fluid rheological properties [8]. We have furthermore shown that particle aggregation is a fast process even when the particles are initially remote from each other and that the formed clusters settle with velocities that are large compared to the one of an isolated particle [9].
One therefore expects a strong influence of particle aggregation on the settling of suspensions in shear-thinning fluids even at small particle volume fractions.

The typical settling behavior we have observed in the suspensions is displayed in the photographs of Figure 1. The particles that are initially homogeneously dispersed (a) swiftly organize into vertical structures while a sharp interface separates the suspension from the particle-free supernatant (b). This interface soon blurs as the particle concentration quickly decreases within the suspension (c and d). The vertical structures later fade away (e to g) and the few remaining particles are very long to settle down (h). This settling behavior utterly differs from the one in Newtonian fluids. Let us remind that in such fluids monodisperse suspensions exhibit a net sedimentation front that separates the homogeneous suspension from the particle-free supernatant. This front moreover moves down at a constant velocity that depends on the Stokes' velocity and the particle volume fraction of the suspension $[11,12]$.

The present work aims at quantitatively characterizing both the settling kinetics and the formation of structures within suspensions in shear-thinning fluids. The paper is organized as follows: in Section 1 we present the preparation of the suspensions as well as the method of measurement. Section 2 is devoted to the fluid characterization and Section 3 to the settling kinetics through the study of the variations of the local particle volume fraction within the suspension. Finally, Section 4 deals with the particle-rich structures that form during the settling.

\section{MATERIALS AND METHODS}

\subsection{Fluids and Particles}

The fluids are Xanthan aqueous solutions. Xanthan is a semirigid polysaccharide. The sample we use (Sigma) is of high molecular weight $\left(\approx 210^{6} \mathrm{~g} / \mathrm{mol}\right)$. Xanthan solutions are prepared by slowly adding the polymer to the solvent constituted by deionised water containing sodium chloride $(10 \mathrm{~g} / \mathrm{l})$ and sodium azide $(0.2 \mathrm{~g} / \mathrm{l})$. The presence of sodium chloride contributes to screen the charges borne by the polymer and sodium azide is a biocide. The solutions are strongly stirred for $48 \mathrm{~h}$ before use in order to completely dissolve the polymer and they are further kept refrigerated to ensure preservation. The polymer concentrations $c_{P}$ of the different solutions varies from 750 to $3000 \mathrm{ppm}$, they all correspond to the semi-dilute regime range.

The particles are made of a composite of zirconia and silica ceramic (Zirblast, SEPR, France). They were chosen for their high density $\left(\rho_{s}=3.76 \mathrm{~g} / \mathrm{cm}^{3}\right)$ and for the good optical contrast they present with the fluid. Their sphericity and absence of macroscopic roughness have been checked using electronic microscopy and their radius is $a=47.5 \pm 2.5 \mu \mathrm{m}$. 


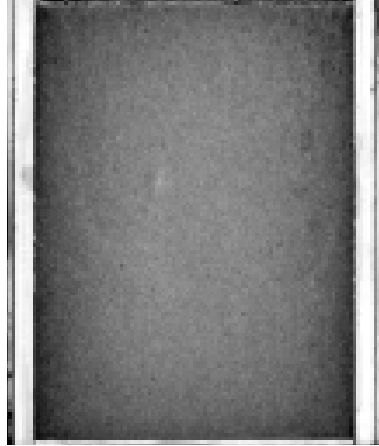

a) $t=0 \mathrm{~s}$

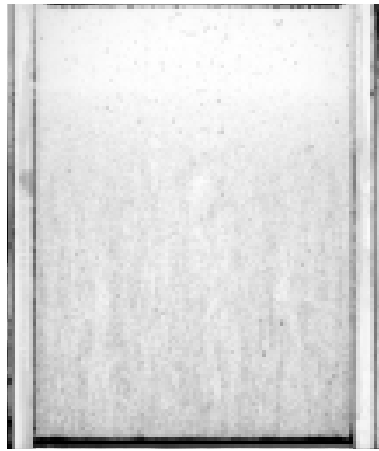

e) $t=400 \mathrm{~s}$

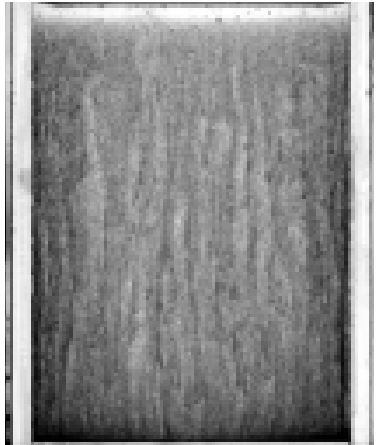

b) $t=52 \mathrm{~s}$

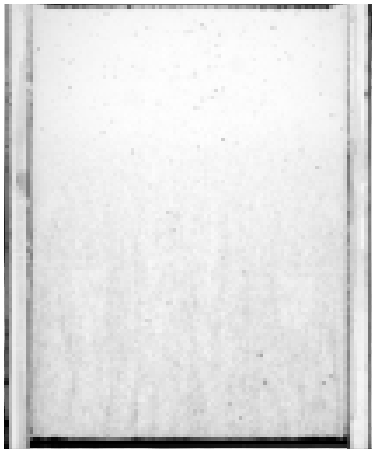

f) $t=600 \mathrm{~s}$

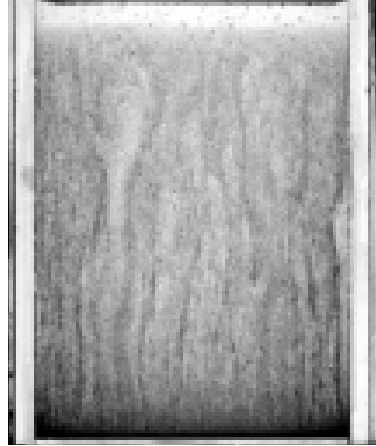

c) $t=100 \mathrm{~s}$

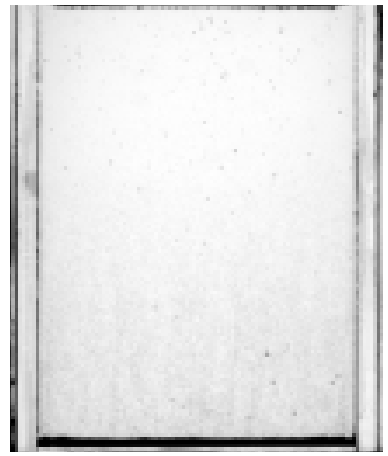

g) $t=800 \mathrm{~s}$

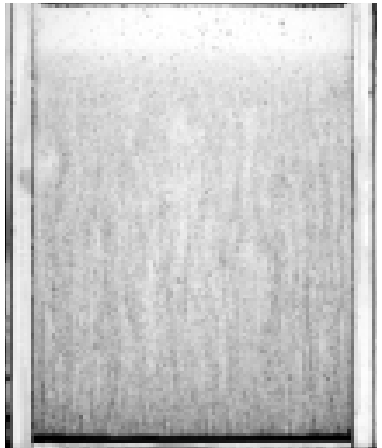

d) $t=200 \mathrm{~s}$

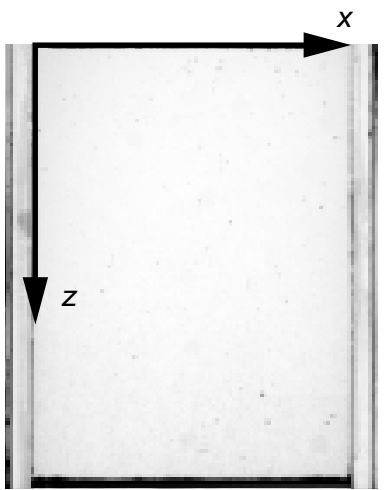

h) $t=1340 \mathrm{~s}$

Figure 1

Settling of a suspension in a shear-thinning fluid at different successive times. The cell is of rectangular section (length $120 \mathrm{~mm}$ ) and is lighted from its back, the particles appearing as black. At time $t=0$ (a), the suspension is homogeneous. The particle concentration quickly decays as vertical dark structures corresponding to concentrated zones appear (b to d). The structures later fade away (e to $f$ ) and the few remaining suspended particles are very long to settle down ( $\mathrm{g}$ to $\mathrm{h}$ ).

In order to study both the influence of the polymer concentration and the particle volume fraction on the settling behavior, experiments have been conducted in all Xanthan solutions at the fixed volume fraction $\Phi=1 \%$ and in the solution of polymer concentration $c_{P}=1500 \mathrm{ppm}$ for different values of $\Phi$ i.e. $0.3 \%, 0.5 \%, 1 \%, 2 \%, 3 \%, 5 \%$ and $7 \%$.

\subsection{Experimental Set Up}

The settling cell is of rectangular section and made of Plexiglas. Its useful height is $200 \mathrm{~mm}$, its inner width $d=$ $12 \mathrm{~mm}$ and inner length $l=120 \mathrm{~mm}$. Prior to each settling experiment the suspension is mixed using a magnetic agitator and then poured into the cell. The total suspension height is $140 \mathrm{~mm}$. The cell is sealed with a Plexiglas cap and the suspension is further mixed by manually agitating the cell. The presence of a large air bubble ensures an effective homogenization and thus a reproducible initial state of the suspension.
The cell is placed with its largest side (i.e. length to height plane) facing a light source that has been designed to provide a homogeneous lighting. Note that the intensity profile along the cell filled with fluid nevertheless presents both a vertical and horizontal curvature due in particular to light reflections on the cell sidewalls.

The settling process is recorded through a digital 12-bit cooled CCD camera (CoolSnap fx, Roper Scientific, United States) that takes views of the length to height plane of the cell. The cell being lighted from the back, the particles appear as black as in Figure 1. With the adopted magnification, the length of the cell corresponds to about 800 pixels. In the following, we use the spatial coordinates $x$ and $z$ that are defined in Figure $1(\mathrm{~h})$. Note that the origin of the $z$-axis is taken at the air/suspension interface. Each film is constituted by 340 images, the time interval between 2 images varying from 5 to $15 \mathrm{~s}$ according to the duration of the experiment.

Finally let us note that we have checked the experimental reproducibility by performing four times each experiment 
corresponding to given particle volume fraction and polymer concentration. Similar results have been found in each case, showing in particular that the initial state of the suspensions is reproducible and thus confirming the efficiency of the mixing process.

\subsection{Analysis of the Experiments}

The video images of the settling suspensions have been analyzed in two different ways, one giving the mean particle volume fraction over the length of the cell, $\varphi(z, t)$ and the other the spatial distribution of the vertical columns that appear during the settling.

To obtain $\varphi(z, t)$ from the luminous intensity of the video images we proceed as follows: first, the intensity along the cell length, $I(x)$, is extracted at given values of height $z$ and time $t$ (see Fig. 1 for the definitions of spatial coordinates $x$ and $z$ ). As pointed out in the preceding subsection, $I(x)$ presents a curvature; this curvature is well described by a third order polynom in $x$ such that:

$$
I(x)=a_{0}+a_{1} x+a_{2} x^{2}+a_{3} x^{3}+\delta I
$$

$\delta I(x)$ being the intensity fluctuations.

The mean value of the intensity over the cell length is then characterized by the calculated coefficient $a_{0}$. The corresponding mean volume fraction $\varphi(z, t)$ is further calculated from $a_{0}(z, t)$ using a model that takes into account both the attenuation of the transmitted intensity due to the particles and the reflections on the particle surfaces. Let us briefly give the principles of this model that is fully detailed elsewhere [10]. We assume that each particle that receives the luminous intensity $I$ reflects the intensity $\alpha I$. If we denote as $y$ the spatial coordinate along the width of the cell, the variation of the luminous intensity $I(y)$ that propagates toward increasing $y$ by unit of surface is therefore given by:

$$
\frac{d I}{d y}=-\frac{1}{\lambda}\left(I-\alpha I_{r}\right)
$$

where $\lambda$ depends on the volume fraction and on the particle radius such that $1 / \lambda=3 \varphi / 4 a$ and $I_{r}$ is the reflected luminous intensity, that propagates toward decreasing $y$.

A similar differential equation can be established for $I_{r}$. The resolution of both these equations finally yields for the luminous intensity in $y=d$ averaged over the cell length, i.e. for $a_{0}$ :

$$
\frac{a_{0}}{I_{\mathrm{inc}}}=\frac{(\beta+1) e^{\beta d / \lambda}+(\beta-1) e^{-\beta d / \lambda}}{\beta+1+(\beta-1) e^{2 \beta d / \lambda}} e^{-2 \beta d / \lambda}
$$

where $I_{\text {inc }}$ is the incident light and $\beta=\sqrt{1-\alpha^{2}}$.
The validity of Relation (3) has been checked using the images of the homogeneous suspensions (i.e. at $t=0$ ) for which the volume fraction is $\Phi$ within the whole cell. A good agreement has been found with a coefficient $\alpha=0.92$, which is consistent with the fact that particles are very white and are therefore expected to greatly reflect light. Note that according to Equation (3), the larger the volume fraction, the more sensitive are the variations of $\varphi$ to the variations of $a_{0}$ : the error done on the volume fraction is therefore larger at large volume fractions. The error however remains small (less than $15 \%$ ) for $\varphi<0.1$. At very small volume fraction, $\varphi<0.0003$, the determination of $\varphi$ also becomes inaccurate since it is very sensitive to the value of $I_{\text {inc }}$. We measured it at the end of the settling process and it thus is sensitive to possible defects of the cell walls or particles stuck on them.

The analysis of the spatial distribution of the particle-rich columns is performed as follows: the luminous contrast of the video images is first improved by averaging the intensities of ten successive images and applying an enhancing filter to the resulting image. Intensity profiles along the cell length are then extracted and averaged over a height of $10 \mathrm{~mm}$. The columns being vertical, this spatial averaging further improves the contrast without altering the data. The positions of the particle-rich zones that appear as dark are then deduced from the positions of the local minima of the intensity profiles.

\section{FLUID CHARACTERIZATION}

\subsection{Rheology}

The viscometric measurements have been carried out using two rheometers equipped with a concentric cylinder apparatus: an imposed-stress rheometer (StressTech, ReoLogica Instruments $A B$, Sweden) and an imposed-strain rheometer (Low Shear, Contraves). All the viscometric measurements have been performed at $25.0 \pm 0.1^{\circ} \mathrm{C}$.

The variations of the steady-state viscosity $\eta$ as a function of shear rate of the Xanthan solutions are shown in Figure 2. All Xanthan solutions present similar properties: at small shear rates, the viscosity is constant (Newtonian plateau) whereas for $\dot{\gamma} \geq \dot{\gamma}_{0}$ the solutions are strongly shear-thinning and the variations of viscosity are well described using a power-law model:

$$
\eta=m \dot{\gamma}^{n-1}
$$

The parameters $m$ and $n$ as well as the critical shear rate $\dot{\gamma}_{0}$ depend on the polymer concentration. They have therefore been measured for each solution and are listed in Table 1.

Finally, it is worthwhile to note that Xanthan being a semi-flexible polymer, Xanthan solutions present very small normal stress differences in particular compared to the ones 


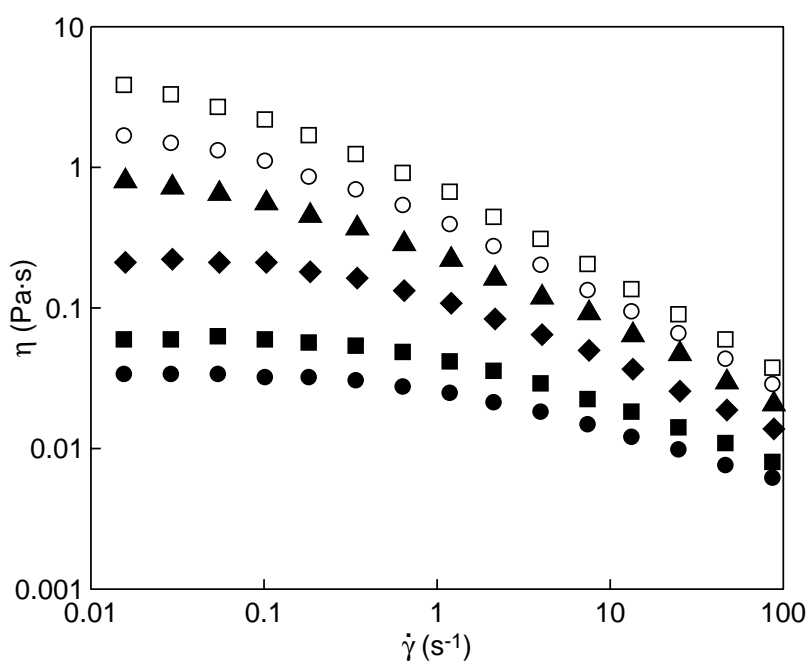

Figure 2

Steady-state viscosities as a function of shear-rate of the Xanthan solutions listed in Table 1 . The polymer concentration increases from the bottom to the top of the figure. The measurements have been performed at the temperature of $25^{\circ} \mathrm{C}$.

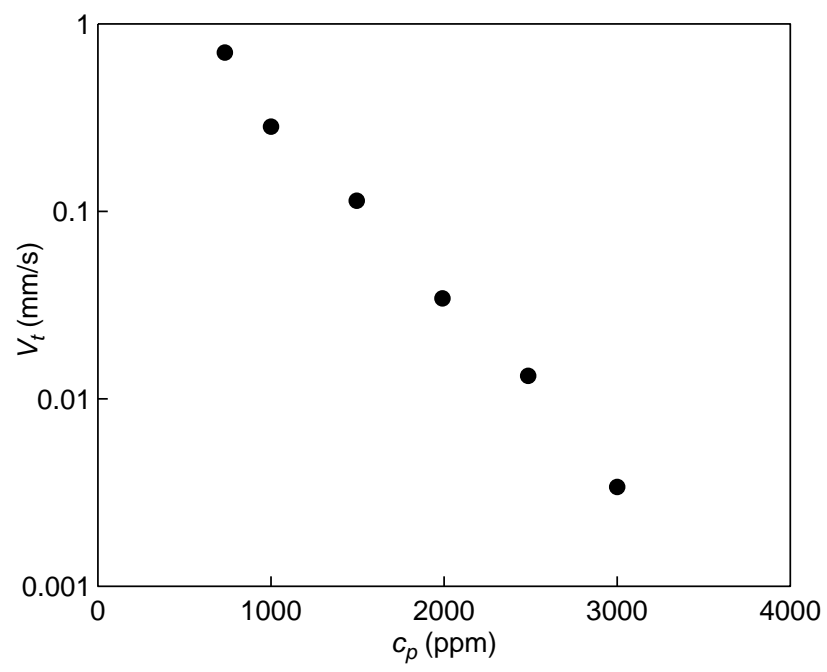

Figure 3

Steady velocity of a single particle (radius $a=50 \mu \mathrm{m}$ ) as a function of the Xanthan concentration of the suspending fluid. Each value corresponds to the average velocity over five different particles. exhibited by usual polymeric fluids that are flexible polymer solutions [13-15]. The effect of normal stresses in the Xanthan solutions we use can thereby be neglected compared to the influence of shear-thinning.

\section{TABLE 1}

Rheological characteristics of the different Xanthan solutions. $\dot{\gamma}_{0}$ is the shear-rate above which the variations of the viscosity are described using Equation (4) and $m$ and $n$ are the corresponding parameters.

\begin{tabular}{c|c|c|c}
\hline Polymer concentration $c_{P}(\mathrm{ppm})$ & $m(\mathrm{SI})$ & $n$ & $\gamma_{0}\left(\mathrm{~s}^{-1}\right)$ \\
\hline 750 & $2.810^{-2}$ & 0.67 & 1 \\
1000 & $4.710^{-2}$ & 0.62 & $610^{-1}$ \\
1500 & $1.310^{-1}$ & 0.54 & $310^{-1}$ \\
2000 & $2.610^{-1}$ & 0.52 & $210^{-1}$ \\
2500 & $4.110^{-1}$ & 0.42 & $510^{-2}$ \\
3000 & $6.910^{-1}$ & 0.36 & $310^{-2}$ \\
\hline
\end{tabular}

\subsection{Settling of an Isolated Particle}

Preliminary settling experiments have been conducted in order to determine $V_{\mathrm{t}}$, the settling velocity of an isolated particle within the different Xanthan solutions. To that extent, we have followed the motion of single particles released along the axis of a cylindrical cell whose dimensions are large compared to the particle radius. For each polymer solution, the velocity $V_{t}$ has been determined by averaging the velocities of five different particles. The results are shown in Figure 3 as a function of the polymer concentration. When $c_{P}$ increases, $V_{t}$ strongly decreases (of about 3 orders of magnitude for $c_{P}$ ranging from 750 to $3000 \mathrm{ppm}$ ).

From the data of Figure 3 one can estimate the generalized particle Reynolds number which is classically defined in a shear-thinning fluid such that:

$$
\operatorname{Re}=\frac{\rho_{f} V_{t}^{2-n}(2 a)^{n}}{m}
$$

where $\rho_{f}$ is the fluid density. In all the presented experiments Re remains smaller than $510^{-3}$.

\section{VARIATIONS OF THE PARTICLE VOLUME FRACTION}

As pointed out in the Introduction, the settling behavior in a shear-thinning fluid is characterized by a variation of the particle volume fraction along the height of the cell. Note that it is contrary to what is observed in a Newtonian fluid in which a sedimentation front separates the particle-free supernatant from the suspension whose mean volume fraction is equal to the initial one. In order to characterize the settling kinetics in the Xanthan solutions, we have therefore studied the variations of the mean volume fraction over the cell length, $\varphi(z, t)$ The results are discussed in the present Section. 


\subsection{Variations with Time at a Given Height}

Figure 4 shows an example of the temporal variations of $\varphi$ at three different heights in the cell. Three different regimes can be identified whatever the height: at short times, the volume fraction is either equal or larger than its initial value $\Phi$; at intermediate times, $\varphi$ becomes smaller than its initial value and further strongly decreases. Finally, $\varphi$ keeps decreasing at long times but in a much slower way. Two characteristic times can be defined: we denote as $t_{i}$ the first time when $\varphi$ becomes smaller than its initial value. At intermediate times, for $t \geq t$, the decrease of $\varphi$ is well described at all heights by an exponential decay such that:

$$
\varphi(t)=\Phi \exp \left[-\frac{t-t_{i}}{t_{c d}}\right]
$$

where $t_{c d}$ is the characteristic decay time of the volume fraction (see Fig. 4).

We have systematically determined the times $t_{i}$ and $t_{c d}$ by fitting the experimental variations of the volume fraction according to Equation (6). A good agreement between the experimental decrease of $\varphi$ and Equation (6) has been observed in each case as long as the condition $\varphi>\Phi / 10$ is fulfilled. Smaller values of $\varphi$ correspond to the third settling regime that will be described in Section 3.3. Note that for $t<t_{i}$ two different behaviors emerge according to the distance from the interface as well as to the initial volume fraction $\Phi$ : for small volume fractions $(\Phi<2 \%)$ and at any height or for larger volume fractions and close to the interface, $\varphi$ remains constant during $t_{i}$. This behavior is for instance observed in the case of Figure 4 that corresponds to $\Phi=3 \%$ and at distances of 20 and $80 \mathrm{~mm}$ from the interface. On the contrary, at larger initial volume fractions and far enough from the interface, $\varphi$ can first increase to later decrease during $t_{i}$. The latter behavior for instance occurs for $z=120 \mathrm{~mm}$ in the case of Figure 4. Note that the polymer concentration of the suspending fluid (1500 ppm in the case of Fig. 4) has no influence on the kind of behavior during $t_{i}$.

The variations of $t_{i}$ and $t_{c d}$ as a function of height are shown in Figure 5 for $\Phi=3 \%$. The time $t_{i}$ is constant within the experimental uncertainty along the upper part of the cell whereas it increases with $z$ at larger distances from the interface. The time $t_{i}$ is interpreted as the time needed by the particles to form aggregates; during $t_{i}$ and close enough to the interface, the mean particle volume fraction remains constant whereas when $t>t_{i}$, the suspension is structured and the phase of rapid settling starts. The larger values of $t_{i}$ in the lower part of the cell could be related to the sediment formation. This build up could affect the settling process in decreasing the velocity and therefore lead to an increase of the mean volume fraction in the lower part of the cell. In

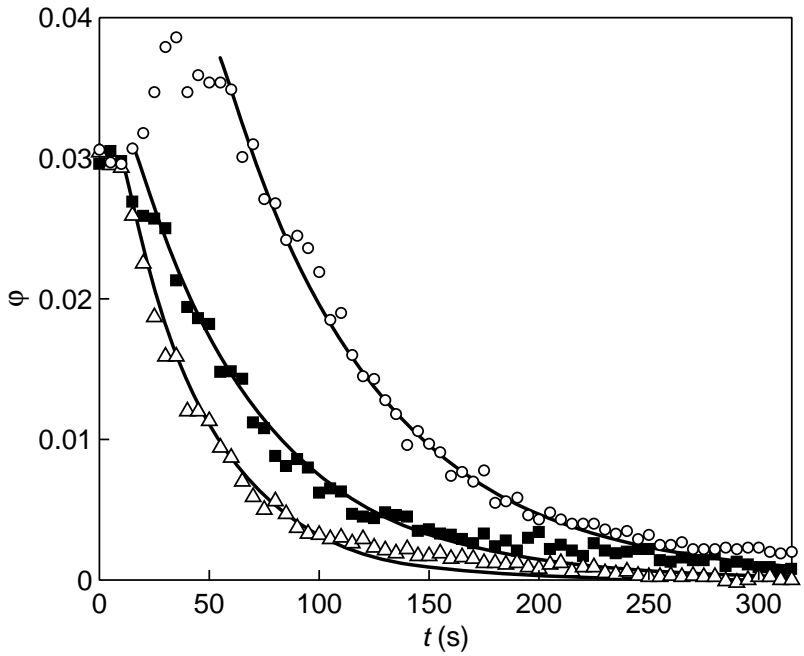

Figure 4

Mean volume fraction over the cell length as a function of time and at three different heights from the air/suspension interface: $z=20 \mathrm{~mm}$ (triangles), $80 \mathrm{~mm}$ (squares) and $120 \mathrm{~mm}$ (circles). The polymer concentration of the suspending fluid is $1500 \mathrm{ppm}$ and the initial volume fraction $3 \%$. The decrease at intermediate times of $\varphi$ is well described at each height using Equation (6) (full lines).

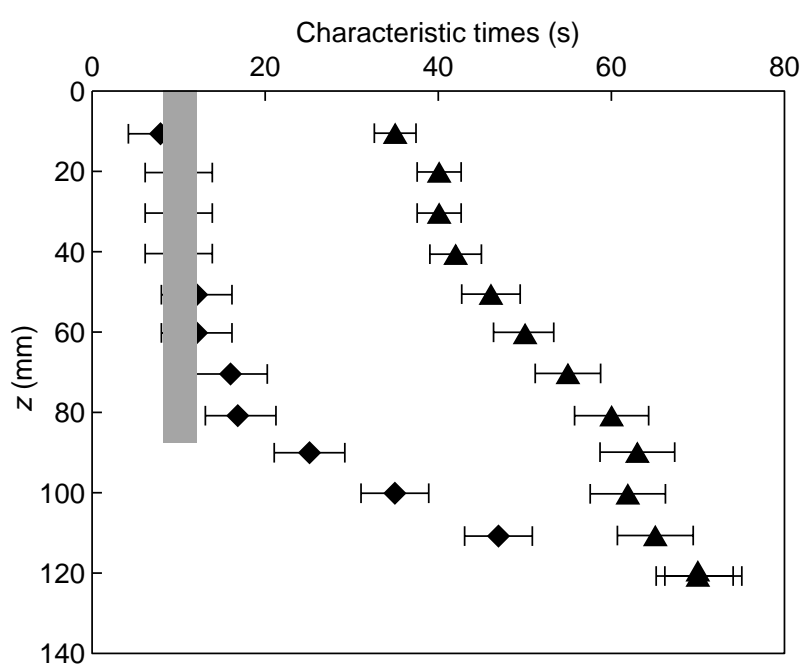

Figure 5

Characteristic times $t_{i}$ (diamonds) and $t_{c d}$ (triangles) deduced from the experimental variations of $\varphi$ along the suspension height. The polymer concentration of the suspending fluid is $1500 \mathrm{ppm}$ and the initial volume fraction $3 \%$. Time $t_{i}$ remains constant from the top to about the mid-height of the suspension and further increases when approaching the bottom of the cell. The structuration time $t_{s}$ is defined as the constant value of $t_{i}$ at smaller heights (grey area). 
order to study the formation of structures we define a more relevant parameter that we denote as $t_{s}$ and that corresponds to the value of $t_{i}$ at small heights as shown in Figure 5.

The time $t_{c d}$ that characterizes the fast settling within intermediate times also varies with $z$ (Fig. 5): the farther away from the interface, the larger is $t_{c d}$ and therefore the slower is the settling process. In the following, we study the influence of the particle volume fraction and polymer concentration on the structuration time as well as a on the characteristic decay time whose value is taken at $z=80 \mathrm{~mm}$.

\subsection{Variations of the Characteristic Times with $\Phi$ and $c_{p}$}

Figure 6 shows the variations of the times $t_{\mathrm{s}}$ and $t_{c d}$ with the polymer concentration. Both times increase exponentially with $c_{P}$. In Figure 6 are also displayed the variations of the characteristic particle time $t_{p}$, i.e. the time needed by an isolated particle to move over its diameter $t_{p}=2 a / V_{\mathrm{t}}$. Both times $t_{s}$ and $t_{c d}$ are larger by about two orders of magnitude than $t_{p}$ and exhibit the same variations with $c_{P}$ as $t_{p}$. In other terms, the distances $V_{t} t_{s}$ and $V_{t} t_{c d}$ are constant whatever the polymer concentration. This last result is consistent with previous ones obtained in the case of a few particles that have shown that the particle velocities in different Xanthan solutions collapse into a single curve provided they are normalized by the velocity of a single particle in the corresponding fluid [8].

Figure 7 shows the variations of $t_{s}$ and $t_{c d}$ as a function of the particle volume fraction of the suspension and at a fixed polymer concentration. Both times decrease with $\Phi$ indicating that the more concentrated is the suspension, the more efficient is the settling. This result is particularly remarkable since it is contrary to what is observed in Newtonian fluids. In such fluids, the velocity of the sedimentation front actually decreases when the volume fraction is increased. Note however that the times $t_{s}$ and $t_{c d}$ are characteristic of the first and second settling regimes and do not reflect the time needed for the total completion of settling. In the case of the characteristic decay time, $t_{c d}$, a plateau seems to be reached for large values of $\Phi$, further investigations are however needed to confirm this result. The structuration time $t_{s}$ keeps decreasing even at large volume fractions and as shown in Figure 7, its decay scales as $1 / \Phi$.

This scaling can be understood by recalling that $t_{s}$ is the time needed for the particles to form aggregates. The time needed for a particle to form a cluster mainly corresponds to the time to move toward its closest neighbors in the vertical direction. The mean number of particles in a cylindrical volume directed in the vertical direction varying as $\Phi$, the mean separation distance between particles in this volume scales as $1 / \Phi$. The time to form a two-particle cluster is therefore
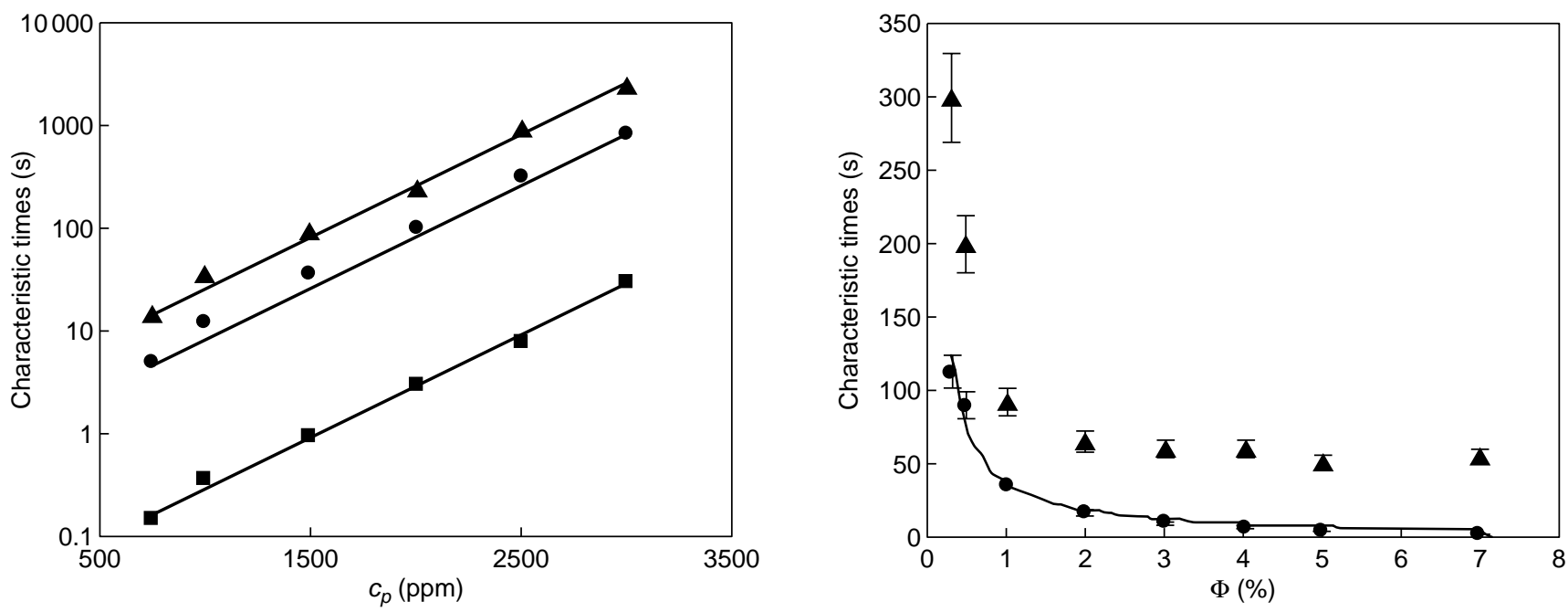

Figure 6

Times $t_{s}$ (circles), $t_{c d}$ (triangles) and $t_{p}$ (squares) as a function of the polymer concentration of the suspending fluid. $t_{s}$ and $t_{c d}$ are deduced from the experimental variations of $\varphi$ and $t_{p}$ is the time needed by an isolated particle to mover over its diameter. All three times exponentially increase with $c_{P}$ (full lines) and their increase is characterized by the same constant. The particle volume fraction is $\Phi=1 \%$.

\section{Figure 7}

Times $t_{s}$ (circles) and $t_{c d}$ (triangles) as a function of the initial particle volume fraction. Time $t_{s}$ decreases as $1 / \Phi$ (full line). The polymer concentration is $c_{P}=1500 \mathrm{ppm}$. 
expected to also vary as $1 / \Phi$ which well corresponds to the experimental observation.

We have shown in this subsection that the influence of the polymer concentration could be simply described by taking into account the velocity of a single particle in the corresponding fluid and that the efficiency of the settling increases with the volume fraction. Both results are valid within short and intermediate times, let us now focus on the phenomena involved at long times.

\subsection{Settling Kinetics at Long Times}

As pointed out in what precedes, the completion of settling in the shear-hinning fluids is a very slow process. Although the number of particles decreases very quickly during the first two phases of settling, the time needed for a complete separation of fluids and particles can be longer than in a Newtonian fluid due to the behavior at long times. This longer separation time is in particular observed in the lower part of the cell. The sluggish sedimentation at long times can be attributed to two effects. Since the non-Newtonian interactions are effective only for particles separated by distances smaller than a critical one, one can invoke the large separation distances between particles once most of the particles have settled i.e. at the end of the second settling regime. We have however observed that the third regime does not start for a given value of the volume fraction but rather for a given value of the ratio $\varphi / \Phi($ i.e. $\varphi / \Phi$ of the order of 0.1 ) whatever the initial particle volume fraction which is not consistent with the invoked argument. The slow settling regime could also be attributed to a wall effect. It has been shown that in shear-thinning fluids the particles are attracted toward the cell walls while settling [4]. We observe this effect in the Xanthan solutions since the sediments formed at the end of settling present an excess of particles along the cell walls. The remaining particles during the last regime could therefore correspond to particles moving slowly along the cell walls. Further investigation is however needed to confirm this assumption.

In what precedes, we have proposed a description of the settling kinetics and shown that owing to the development of vertical structures within the suspensions, the beginning of the settling is much faster than in a Newtonian fluid. The following Section is devoted to the analysis of the spatial distribution of these structures.

\section{VERTICAL STRUCTURES}

As shown in the preceding analysis, the rapid decrease of the particle volume fraction coincides with the formation of the particle-rich columns; these structures are therefore fully developed for $t>t_{s}$. Since their disappearance corresponds to the slow settling regime, we have studied the structures within intermediate times. We have observed that while the positions of the columns within the cell length could evolve in time, their total number remains constant. The number of columns is furthermore independent of the height $z$ provided $z$ is not too small since the structures quickly disappear when close to the interface. As a consequence, the results we present on the total number of columns at time $t=t_{s}$ and height $z=110 \mathrm{~mm}$ are valid within the whole rapid settling regime and at all heights larger than about $30 \mathrm{~mm}$.
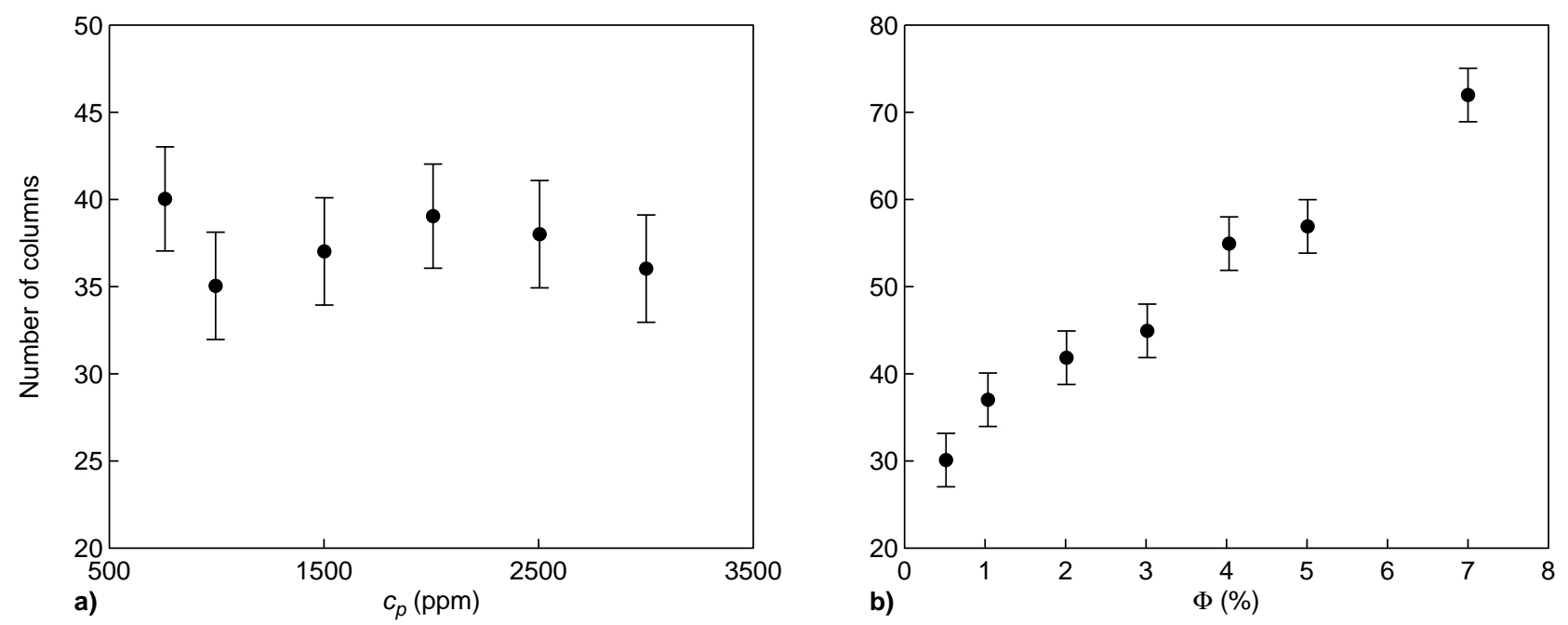

Figure 8

Number of vertical columns at time $t_{s}$ as a function of (a) the polymer concentration of the suspending fluid (for $\Phi=1 \%$ ) and (b) the initial particle volume fraction (for $c_{P}=1500 \mathrm{ppm}$ ). The number of columns is measured at a height $z=110 \mathrm{~mm}$. 


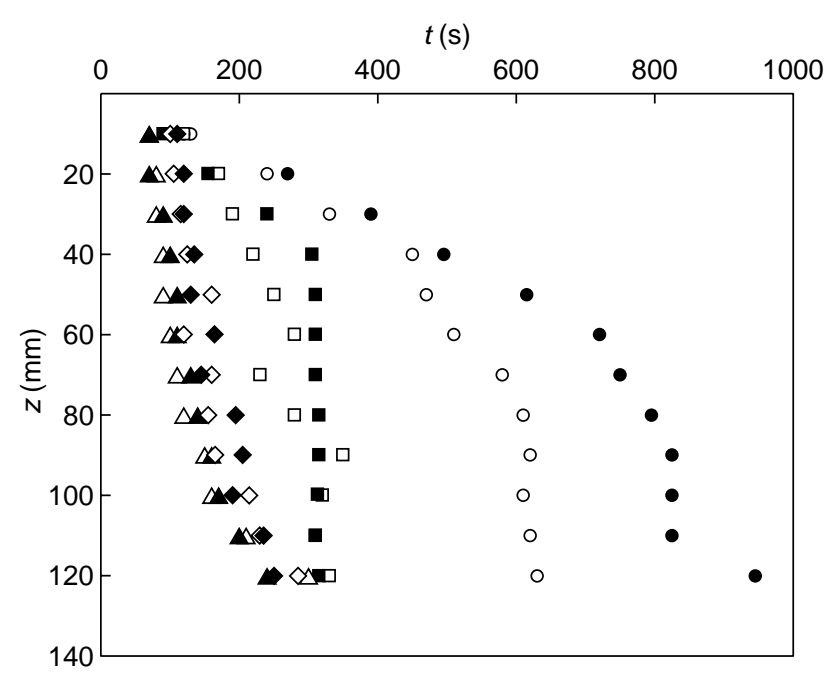

Figure 9

Position as a function of time of iso-volume fraction lines for different initial particle volume fractions and for a polymer concentration of $1500 \mathrm{ppm}$. The iso-volume fraction lines correspond to a particle volume fraction of $\Phi / 10$. From the left to the right of the figure, $\Phi$ decreases from 7 to $0.3 \%$. The data are deduced from the experimental variations of $\varphi$.

Figure 8 displays the variations of the number of vertical columns as a function of the polymer concentration (a) and the particle volume fraction (b). The number of columns remains constant whatever the polymer concentration which is consistent with previous observations on the settling kinetics: since the change of polymer concentration only induces an acceleration or a slowing down of the settling process proportionally to the velocity of a single particle, no effect on the formation of structures is expected. On the contrary, the number of columns increases with the initial particle volume fraction as shown in Figure 8.

\section{CONCLUSION}

We have presented quantitative results on the settling of monodisperse suspensions in shear-thinning fluids. The reported behaviors and in particular the development of nonhomogeneous structures due to particle aggregation are in agreement with previous works performed in polymeric fluids. The study of the temporal variations of the effective particle volume fractions has shown that the settling process goes through three phases. The suspension first remains homogeneous at large scales while particle aggregation occurs at smaller scales. Once the particles aggregated, particle-rich columns form in the direction of gravity and the particle volume fraction decays exponentially at all heights. The number of these columns remains constant with time and the distance between columns is high compare to the particle diameter. Note that the light attenuation technique being mainly sensitive to the bulk of the suspension outside the columns, the decay with time of the volume fraction is related to the decrease of the amount of particles in such areas. The particles seem to be "pumped" out from the bulk to the columns. The third phase corresponds to a very slow settling process that demands further investigation. We have systematically studied the times characterizing the first two phases as a function of the polymer concentration and initial particle volume fraction. One of the most striking results we have obtained is that the efficiency of settling during these phases is improved by increasing the particle volume fraction. This effect is illustrated in Figure 9 that displays the positions as a function of time of iso-volume fraction lines. The results are shown for different initial volume fractions $\Phi$ and in each cases, the iso-volume fraction corresponds to $\Phi / 10$. Note that a similar representation in a Newtonian fluid would lead to a linear variation with time of the position of the iso-volume fraction (that is included in the sedimentation front). In the non-Newtonian case, whereas at small volume fractions the behavior of the iso-volume fraction lines remains similar to the one of a single particle, the displacements of theses lines become very fast at larger volume fractions indicating a much more efficient settling process. This property is indeed opposite to what is found in Newtonian fluids in which the sedimentation front moves slower when $\Phi$ is increased; it moreover is of practical importance for the industrial processes involving settling in shear-thinning fluids such as the transport of particles by drilling fluids. Although some points such as the behavior at long times are still open matters, the characterization of the settling kinetics we propose together with the study of the structures that appear within the suspensions constitutes a first step toward the understanding of sedimentation in fluids of complex rheology.

\section{ACKNOWLEDGEMENTS}

We thank A. Audibert, H. Li and M. Moan for their interest in the subject. Laboratoire "Fluides, Automatique et Systèmes Thermiques" is a laboratory of Universities Paris VI and Paris XI, and of CNRS (UMR 7608).

\section{REFERENCES}

1 Chhabra, R.P. (1993) Bubbles, Drops and Particules in Non-Newtonian Fluids, CRC Press, Boca Raton, FL.

2 Riddle, M.J., Narvaez, C. and Byrd, R.B. (1977) J. Non-Newtonian Fluid Mech., 2, 23.

3 Liu, Y.J. and Joseph, D.D. (1993) J. Fluid Mech., 82, 565.

4 Joseph, D.D., Liu, Y.J., Poletto, M. and Feng, J.J. (1994) J. Non-Newtonian Fluid Mech., 54, 45.

5 Gheissary, G. and Van den Brule, B.H.H.A.(1996) J. NonNewtonian Fluid Mech., 67, 1. 
6 Allen, E. and Uhlherr, P.H.T. (1989) J. Rheol., 33, 627.

7 Bobroff, S. and Phillips, R.J. (1998) J. Rheol., 42, 1419.

8 Daugan, S., Talini, L., Herzhaft, B. and Allain, C. (2002) Eur. Phys. J. E, 7, 73.

9 Daugan, S., Talini, L., Herzhaft, B. and Allain, C. (2002) Eur. Phys. J. E, 9, 55.

10 Daugan, S. (2002) Sédimentation de particules isolées et de suspensions en milieu rhéofluidifiant. PhD Thesis, University Paris VI .

11 Batchelor, G.K. (1972) J. Fluid Mech., 52, 245.
12 Richardson, J.F. and. Zaki, W.M. (1954) Trans. Inst. Chem. Eng., 32, 35.

13 Allain, C., Cloître, M., Perrot, P. and Quemada, D. (1993) Eur. J. Mech. B/Fluids, 12, 175.

14 Zirnsak, M.A., Boger, D.V. and Tirtaatmadja, V. (1999) J. Rheol., 43, 627.

15 Barnes, H.A., Hutton, J.F. and Walters, K. (1989) An Introduction to Rheology, Elsevier, Amsterdam.

Final manuscript received in December 2003 